\title{
Un enfoque gerencial de factores críticos para el éxito de los sistemas de información en la Pyme metalmecánica venezolana
}

José Luis Calderón

Amaya

Departamento de Organización y Gerencia, Universidad Nacional Experimental de Guyana jlca1150@gmail.com

\section{Carlos Rodríguez}

Monroy

Escuela Técnica Superior de Ingenieros Industriales, Universidad Politécnica de Madrid crmonroy@etsii.upm.es

\section{Resumen}

Informes oficiales de países en desarrollo señalan, en general, significativas deficiencias en el tratamiento de la información en las pequeñas y medianas empresas (Pyme). Contar con sistemas de información automatizados (SI) es ineludible, pero es más importante que sean exitosos, para lo cual la satisfacción del usuario final es el factor clave que llevará a obtener los beneficios esperados. Los niveles gerenciales y los profesionales de informática deben estar familiarizados con los principales factores relacionados para asegurar su adecuado tratamiento. Este estudio evaluó la satisfacción del usuario final y varios factores críticos de éxito relacionados en una muestra de empresas industriales medianas (Pymi). Para ello, se utilizó uno de los modelos de éxito más reconocidos por la comunidad investigadora del área. Realizados los análisis cuantitativo/cualitativo y comparados los resultados se concluye que el principal factor relacionado con la satisfacción del usuario final es la calidad de la información, lo cual puede ser suficiente para considerar como exitoso un SI; con esto los demás factores quedan en segundo lugar. El beneficio práctico de esta investigación es reflexionar sobre estos factores, contribuir a reforzar la efectividad y calidad de los procesos de desarrollo o adquisición de un SI y reducir su índice de fracasos.

Palabras clave: Pyme metalmecánica, sistemas de información (SI), factores críticos de éxito, satisfacción del usuario final. 


\title{
A managerial approach to key factors for information systems success on Ve- nezuelan metalworking SMEs
}

\begin{abstract}
Official reports from developing countries brought significant shortcomings in the treatment of information to SMEs in general. Having automated information systems (IS) is mandatory, but even more important is that they are successful, which depends heavily on end-user satisfaction as the key that will lead to the expected benefits. Managerial levels and IT professionals should be familiar with the main factors associated with that success, to ensure their adequate treatment. This study evaluated the end-user satisfaction and several critical related success factors, in a sample of medium-sized industrial enterprises SMEs (SMIs), based on two IS critical to any organization, such as payroll and human resource management. For this purpose, one of the success models most recognized by the research community in the area was used. Practical benefits of this research are to call to reflection on these factors, to contribute to strengthen the effectiveness and quality of the development or acquisition process of an SI and to reduce their failure rate.
\end{abstract}

Keywords: metalworking SME, Information systems (IS), critical success factors, end-user satisfaction.

\section{Introducción}

Es bien sabido que el sector de pequeñas y medianas empresas (Pyme) sigue siendo un importante contribuyente económico al desarrollo regional y nacional en cualquier país. Según Doyle (2009), en Europa "existen unos 23 millones de Pyme en la Unión Europea (UE), que representan más del 90\% de todas las empresas y el $57 \%$ del producto interno bruto (PIB) de la UE”. Por otra parte, la Pyme constituye la médula espinal de la economía y el principal responsable de la riqueza y el crecimiento económico. El principal apoyo de la economía europea está en la Pyme (European Commission, 2010).

Estudios recientes del Banco Mundial, del Banco Interamericano de Desarrollo y de la Comisión Económica de las Naciones Unidas para América Latina estiman que entre el $90 \%$ y el $98 \%$ de las unidades productivas en América Latina contribuyen con alrededor del $63 \%$ del empleo y entre un $35 \%$ a un $40 \%$ del producto de la región. Estudios particulares han mostrado cifras similares, lo que indica que el 90\% de la Pyme, en promedio, en países de América Latina proporciona empleo 
aproximadamente al $70 \%$ del mercado de trabajo y contribuye entre un $20 \%$ y un 30\% del PIB (Universia Knowledge, 2010).

Simultáneamente, la globalización sigue presentando nuevos retos, exigiendo así un mejor uso y gestión de las herramientas de tecnologías de la información y telecomunicaciones (TIC). La Pyme invierte poco en SI y TIC y, cuando lo hacen, puede ocurrir que la falta de alineación con la estrategia del negocio origine que estas inversiones sean insuficientes o inadecuadas. La Fundación para el Desarrollo Sostenible en América Latina (Fundes) revela algunas cifras desalentadoras que indican que la Pyme invierte sólo el $2 \%$ de sus presupuestos en SI y TIC. Para algunos expertos, cuando un empresario de la Pyme piensa en el presupuesto para SI y TIC lo equipara con un gasto, no con una inversión; posteriormente, adquiere los SI más económicos o los menos adecuados para el negocio, lo que origina resultados adversos (Universia Knowledge, 2010).

En muchas economías en desarrollo los gobiernos vienen señalando deficiencias de información dentro de la Pyme, como es el caso en Venezuela con las pequeñas y medianas industrias (Pymi), un segmento de gran importancia dentro de la Pyme. Se cree que estas deficiencias, entre otros factores, están afectando el crecimiento potencial del sector debido a la falta de un mayor énfasis en la gestión de la información por parte de estas empresas. A este respecto muchos países están emprendiendo esfuerzos para apoyar el desarrollo de la información en dichas empresas, mediante las TIC (PDESN, 2007). Los gobiernos están promoviendo instituciones dedicadas a su promoción y desarrollo. Así, por ejemplo, en Venezuela se ha creado el Instituto Venezolano para el Desarrollo de la Pymi (INAPYMI). Los ministerios involucrados están convocando a los organismos de ciencia y tecnología, a las universidades y a sus centros de investigación para contribuir a tales fines (PNCTI, 2010).

En Venezuela, el segmento Pymi es especialmente relevante para las industrias básicas nacionales del mineral de hierro, bauxita, alúmina, aluminio, acero, oro y electricidad. Estas Pymi realizan un rol de proveedor clave para las operaciones de las referidas industrias nacionales que constituyen las fuerzas alternativas (no petroleras) para el desarrollo económico del país. Ello ha constituido la razón principal para su selección en este estudio. Otra razón ha sido su capacidad económica para invertir en herramientas de TIC, muy por encima de la del resto de la Pyme en la región y tal vez en Venezuela. Profundizar en la descripción de los productos y servicios proporcionados por este segmento de la Pyme no es un objetivo de este 
documento; es importante señalar que éstos constituyen insumos importantes para las industrias básicas de la nación.

El objetivo de evaluar la satisfacción del usuario final, como reflejo del éxito de los SI (DeLone y McLean, 2003), se ha realizado mediante un análisis cuantitativo para la jerarquización de los principales factores involucrados. Los factores de éxito para ser estudiados son calidad de la información, calidad del SI, calidad de los servicios sobre el SI, uso del SI, rendimiento individual y rendimiento de grupo. Estos factores o variables pertenecen al modelo de DeLone y McLean (D\&M) (1992, 2003), explicado más adelante.

El término usuario final de un SI se refiere a los trabajadores de una organización cuyas tareas dependen fuertemente de la utilización del sistema, especialmente cuando esa dependencia tiene una frecuencia alta. Este estudio responde al hecho de que sin la satisfacción del usuario final el uso de los SI es muy reducido y derivar información interna o externa útil de un SI es con frecuencia difícil. La pregunta de investigación es: ¿cuál es el nivel de satisfacción del usuario final y cuáles son los principales factores que impactan la misma en el segmento estudiado de la Pymi?

La Pyme en los países en desarrollo muestra similitudes en la forma en que invierten en SI y TIC y en cómo utilizan estos medios tecnológicos para operar y administrar sus negocios. Por lo general, éste es el caso de la Pyme en América Latina, donde el concepto y el valor de la información son con frecuencia subestimados (Cornella, 1997; European Commission, 2010). Sobre la base de estos argumentos, se considera que este trabajo aporta cierto conocimiento y algunas guías útiles a la gerencia de la Pyme para la gestión de la información automatizada. Un escenario interesante para el desarrollo sostenible de Venezuela se encuentra en la región de Guayana, la cual posee un rol crítico por acoger a más del $90 \%$ de las industrias básicas de la nación. Por lo tanto, las empresas objetivo del trabajo de campo ha sido integrado por una muestra representativa de la Pymi metalmecánica de Ciudad Guayana, estado Bolívar, Venezuela.

Aspectos de gestión y conductuales, más que los técnicos, constituyen la esencia del estudio; de aquí los términos "enfoque gerencial" en el título de este artículo. Por ello, el proyecto también incluye la recopilación de información adicional sobre el tema para la identificación de puntos débiles y oportunidades en el área de SI y TIC. Otro propósito es que los profesionales informáticos y las universidades encuentren en este trabajo ideas para una mayor cooperación con la Pyme 
en general, que pudiesen traducirse en diseñar mejores prácticas, estrategias, administración y soporte técnico, para el área de los SI y TIC. Se espera así que los resultados también ayuden a la gerencia de este tipo de empresas a identificar y gestionar los factores que pueden conducir a disponer de sistemas de información automatizados exitosos.

En este estudio, la Pymi se clasifica de acuerdo con el Código Industrial Uniforme (CIU) de las Naciones Unidas para este tipo de empresas, como se observa en el cuadro 1 (INE, 2009).

\section{Cuadro 1 \\ Código Industrial Internacional Uniforme}

\begin{tabular}{l|l|l}
\hline & \multicolumn{1}{c}{ Código Industrial Internacional Uniforme - CIIU } \\
\hline División 28 & Fabricación de productos metálicos, excepto maquinaria y equipos \\
\hline & 281 & $\begin{array}{l}\text { Productos metálicos para uso estructural, contenedores, depósitos y } \\
\text { generadores a vapor. }\end{array}$ \\
& 289 & Otros productos metálicos: servicios metalmecánicos. \\
\hline División 29 & Manufactura de maquinaria y equipos N.C.P. \\
\hline & 291 & $\begin{array}{l}\text { Maquinaria de uso general. } \\
\text { Maquinaria de uso especial. }\end{array}$ \\
\hline
\end{tabular}

Fuente: Instituto Nacional de Estadística - Venezuela (INE) 2009

Del mismo modo, el cuadro 2 muestra la clasificación realizada por el INE (2009) por estrato de ocupación, la cual es utilizada en esta investigación para escoger el objeto de estudio. En la muestra sólo se incluyen empresas ubicadas en las capas mediana industria superior y mediana industria inferior de este cuadro. Por lo tanto, se consideraron las empresas con un número de empleados (NE) entre 21 y 100. Cabe mencionar que aunque el NE no es el único parámetro para establecer el tamaño de la Pyme, éste es el único criterio que suministra el INE venezolano para no revelar información considerada confidencial. Además, el NE puede variar según el esquema de clasificación que se utilice para definir cada estrato, así como el país en cuestión. En este caso, la clasificación de industrias proporcionada por el INE se estimó como la más apropiada. 


\section{Cuadro 2}

Clasificación por estrato de ocupación

\begin{tabular}{lcc}
\hline \multicolumn{1}{c}{ Tipo } & Estrato de ocupación & Personal ocupado (PO) \\
\hline Gran industria & 1 & $\mathrm{PO}>100$ \\
Mediana industria superior & 2 & $51 \leq \mathrm{PO} \leq 100$ \\
Mediana industria inferior & 3 & $21 \leq \mathrm{PO} \leq 50$ \\
Pequeña industria & 4 & $5 \leq \mathrm{PO} \leq 20$ \\
\hline
\end{tabular}

Fuente: Instituto Nacional de Estadística, Venezuela (INE) 2009

Rasgos característicos de las TIC tales como la alta velocidad en la captura, procesamiento y entrega de información son factores clave para el éxito, pero al final lo que cuenta realmente de un SI es la calidad de la información de salida y su calidad como herramienta (por ejemplo, su fiabilidad, solidez y facilidad de uso, entre otros). Las TIC deben no sólo apoyar procesos críticos, las funciones administrativas y las tareas que se realizan regularmente, sino también proporcionar información útil y crear ventajas competitivas (Cornella, 1997). Pero no por poseer una mayor inversión en TIC estará una empresa en mejor posición competitiva dentro de su industria. Con el fin de lograr este objetivo es necesario saber utilizar estas herramientas.

\section{Fundamento teórico del estudio}

El modelo de DeLone y McLean (2003), mostrado en la figura 1, es uno de los más reconocidos en la investigación sobre SI desde 1992. Se han agregado, además, algunos aspectos organizacionales relacionados que también son críticos para evaluar la efectividad de los SI (Rai et al., 2002; Wixom et al., 2005). No sólo un número considerable de estudios sobre el tema han utilizado el modelo de D\&M como guía, sino que también algunos de ellos han ampliado el mismo (Medina, 2005; Pitt et al., 1995), con lo que han dado solidez teórica a la presente investigación. Algunos otros trabajos sobre la dimensión de satisfacción del usuario final, lo que ésta implica y sus factores relacionados, se consideran clave para la aplicación y utilidad sostenida de los SI en cualquier tipo de empresas. No obstante, en este estudio, el interés no es puesto en el modelo propiamente, sino en su utilización para analizar los factores mencionados y sus relaciones con el fin de lograr los objetivos propuestos. 
Figura 1

Modelo de éxito de los SI - actualizado

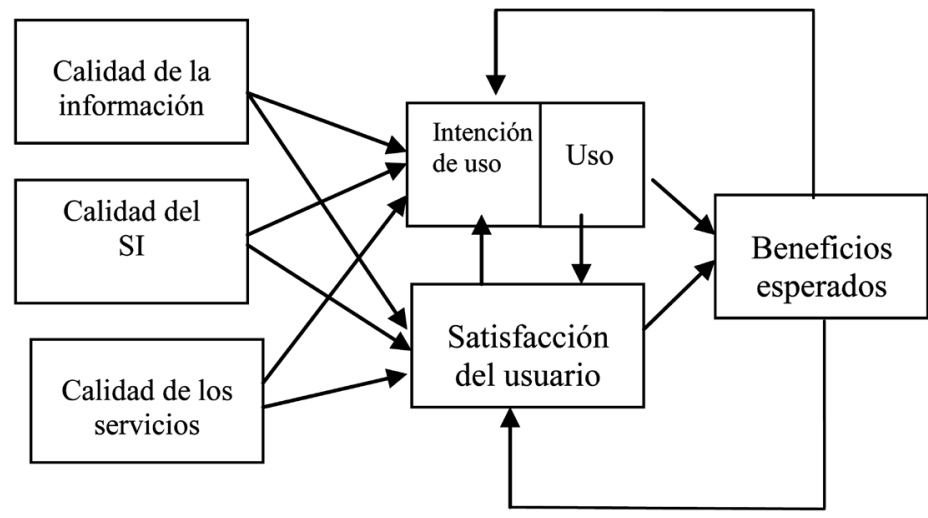

Fuente: DeLone, W. y McLean, E. 2003 adaptado por los autores.

Es por lo anterior que se presenta a continuación una síntesis de los objetivos. Por calidad de la información se entiende la exactitud, relevancia, completitud, oportunidad y consistencia de ésta. La calidad del SI viene dada por su funcionalidad, confiabilidad, facilidad de uso, portabilidad, flexibilidad e integración con otros SI. Este concepto está dirigido, básicamente, a las características técnicas de un SI. La calidad de los servicios hace referencia al apoyo recibido del departamento de SI o de un proveedor externo. El usuario final es su cliente y un soporte deficiente puede dar origen al desencanto y pérdida de éste. Esta dimensión o factor incluye hardware y software actualizado, confiabilidad, servicio rápido y empatía con las necesidades del usuario final.

El uso del SI mide toda interacción del usuario final con el mismo, ya sea consultando información o ejecutando una transacción. El concepto de satisfacción del usuario final es definido por Torkzadeh y Doll (1999) como el grado hasta el cual el SI ayuda al usuario final a crear valor para sus clientes internos [otras áreas de la organización] o externos. Este factor se estima que es el condicionante clave para el éxito de un SI porque implica su utilización y lleva a los beneficios netos esperados. Para DeLone y McLean, los beneficios esperados representan las principales mediciones de éxito de un SI y están conformados, en primer lugar, por el desempeño individual medido en términos de capacidad de decisión, productividad en el trabajo y calidad del trabajo; y en segundo lugar, el desempeño grupal representado por la suma de estos mismos términos en la organización. Sin embargo, este 
estudio se orienta más hacia la evaluación de la satisfacción del usuario final —por lo antes expuesto y por la observación en la práctica de la disciplina- y de cómo ciertos SI con la potencialidad de incrementar significativamente el rendimiento laboral individual y colectivo fueron rechazados por el usuario final por factores estrictamente personales, dando como resultado el fracaso de los mismos.

El uso estratégico de las herramientas informáticas es considerado un factor eficaz para el desarrollo económico (Hummel, 2001), para la generación de ventajas competitivas (Cohen y Asín, 2004) y para el incremento de la productividad (Kotler et al., 2004). Sin embargo, como es el usuario final el principal responsable de la utilización de esta tecnología, dicha utilización está influida por las habilidades y la aceptación de éste, considerando estos rasgos conductuales como un ingrediente necesario para el éxito de tales herramientas (Torkzadeh, 2003). Para comenzar, a menos que el usuario final pueda percibir beneficios de sus SI, la intención de utilizarlos será baja (Shin, 2002). Otro motivo ampliamente aceptado para afirmar que la "satisfacción del usuario final" puede ser una medida determinante del éxito ha sido el hecho de que es el mismo usuario final quien decide en qué medida sus necesidades de información son atendidas por la tecnología (Jiang et al., 2001; Mahmood et al., 2001; Mahmood et al., 2000).

Al mismo tiempo, el usuario final tiene una influencia directa sobre su propia satisfacción, a la hora de aportar las especificaciones y participar en las fases de análisis, diseño y prueba de sus SI, fases en las cuales se gesta la calidad de los últimos. Igual importancia tiene su total compromiso y participación en la fase de implantación para la depuración y el adecuado mantenimiento de los SI.

\section{Estudio empírico}

El modelo DeLone y McLean, como se indicó anteriormente, ha sido utilizado para lograr el objetivo de esta investigación. Se diseñó y aplicó una encuesta a 71 usuarios finales en 45 empresas metalmecánicas de tamaño mediano, aleatoriamente seleccionadas entre los miembros de las asociaciones de empresas industriales metalmecánicas y metalúrgicas (AIMM, 2009) de Ciudad Guayana, Venezuela. Estas asociaciones incluyen más del $90 \%$ de este tipo de empresas en la región, de acuerdo con el ranking de las industrias del Instituto Nacional de Estadística (INE) (cuadro 2), conformando la mejor representación de la población general de Pymi por ser estudiada. Se aplicaron las técnicas estadísticas a los datos organizados, utilizando el software SPSS. Algunos datos descriptivos de los encuestados indican 
que: a) en torno al 50\% tienen educación universitaria, b) el promedio de años de trabajo en la empresa es seis, c) el promedio de años utilizando SI es cuatro, d) el promedio de horas semanales de uso de SI es cinco, e) la media de las edades es de 32 años.

Debido a su significación y complejidad en cualquier organización, las áreas de recursos humanos (RRHH) y sus principales SI (Nómina y Gestión de los RRHH) han sido seleccionadas para el trabajo de campo. Existen tres razones fundamentales para elegir estos SI: 1) todas las empresas estudiadas utilizan, al menos, un SI de nómina idéntico o muy similar, 2) tanto el de Nómina como el de Gestión de los RRHH son SI críticos y complejos que procesan básicamente transacciones y poseen características similares, pero al mismo tiempo adaptados a las particularidades de cada organización y 3 ) son apropiados para futuros análisis.

Con respecto a las técnicas de recolección de datos utilizadas, una encuesta estructurada es aplicada directamente a los usuarios finales de los SI de nómina y de gestión de recursos humanos. Esto se efectuó mediante el uso de un cuestionario con escala tipo Likert de 5 niveles y 62 ítems, de un total de 70 ítems tomados en su mayoría de otros trabajos de investigación similares (IS World Net, 2010; Eencuesta, 2010; Medina, 2005; DeLone y McLean, 2003; Rai et al., 2002; Jiang et al., 2002; Doll y Torkzadeh, 1998; Saarinen, 1996; Bailey y Pearson, 1986). La confiabilidad del mismo fue medida mediante el alfa de Cronbach, arrojando un valor de 0.906, por encima del mínimo aceptable (0.70). Asimismo, el cuestionario fue validado por análisis factorial, conduciendo a la eliminación de sólo 8 ítems de un total de 6 componentes principales.

Otro modelo subyacente a la metodología utilizada es el modelo de Michael Myers $(2010,2009,2007)$ sobre investigación cualitativa en el área de los SI que apoya el enfoque de triangulación (una combinación de métodos cuantitativos y cualitativos). Esta modalidad es representada para este artículo en la figura 2. Myers defiende la idea de que se ha producido un giro en la investigación del área de SI que la aleja del enfoque meramente tecnológico y la reorienta prioritariamente hacia un enfoque de gestión y de organización. Esta tendencia ha dado origen a un creciente interés en la aplicación de métodos de investigación cualitativa. Por lo tanto, una entrevista semiestructurada, validada por un equipo de expertos, se ha utilizado para la recopilación de datos de los informantes clave (alta dirección). Adicionalmente, se obtuvieron comentarios y observaciones de valor agregado de parte de los encuestados. 


\section{Figura 2}

Método de triangulación

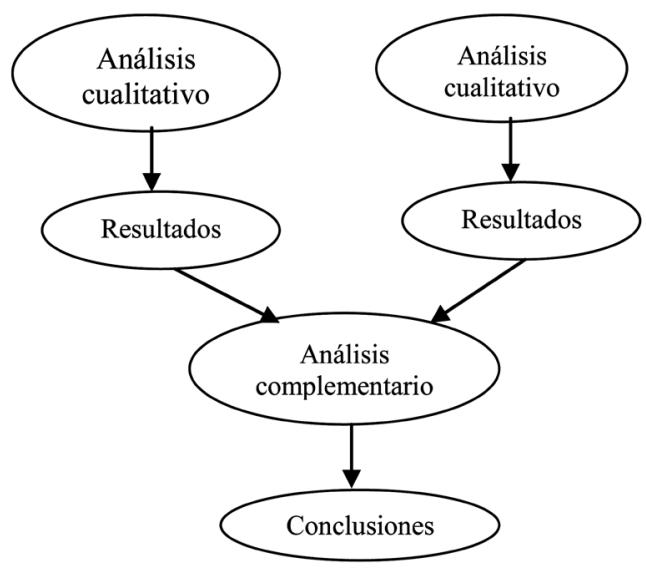

\section{Resultados}

\section{Procedentes del análisis cuantitativo}

Los datos cuantitativos se analizaron utilizando varios métodos estadísticos de orden descriptivo, correlacional y de regresión múltiple, a fin de probar la hipótesis tácita: "cuanto mayor sea la calidad de la información y la calidad técnica del SI, tanto mayor será la satisfacción con el SI y, por ende, los niveles de rendimiento personal y grupal". Las mediciones descriptivas, de correlación y de regresión son usadas para estudiar cómo las variables predictoras interactúan con la dimensión o factor "satisfacción del usuario final", según el modelo de D\&M.

Todos los ítems usados en el cuestionario tienen una puntuación de 1 a 5, donde los valores bajos están próximos a 1 y los valores altos están próximos a 5. Los valores aceptables se consideran aquellos iguales o superiores a 4 . El cuadro 3 muestra los factores del modelo revisado y las respectivas medidas estadísticas. Por tratarse de variables ordinales, la medida más representativa es la mediana y los valores más altos pertenecen a las variables calidad de la información (4.444) y a la calidad del SI (4.330), las cuales tienen también valores elevados en sus respectivas medias. La calidad de la información comienza a mostrar su relevancia como factor determinante, seguido del factor rendimiento individual (4.290) y del factor calidad del servicio (4.000). La satisfacción del usuario final también muestra un valor 
alto (4.370). El análisis refleja que los usuarios finales no fueron capaces de estimar cómo los SI han podido afectar al factor desempeño organizacional o grupal. Muchos de ellos señalaron que sólo podían hablar por sí mismos, marcando así el valor de indecisión de la escala de Likert (valor 3). Se asume que ésta pueda ser la razón para obtener el promedio más bajo (3.571).

No obstante, como se explica en el análisis posterior de los datos cualitativos, se encuentra, por ejemplo, que por el tipo de SI (prácticamente off-the-shelf) instalados en la mayoría de estas empresas la falta de flexibilidad es muy común y ello se traduce en cierta rigidez para el proceso de la información. Esto contribuye a limitar la capacidad de adaptabilidad de los SI a los requerimientos cambiantes de información exigidos por las organizaciones.

\section{Cuadro 3}

\section{Datos descriptivos estadísticos}

\begin{tabular}{lrrr}
\hline Variables & Media & Mediana & Dev. Std. \\
\hline Uso del SI & 4.097 & 4.200 & 0.522 \\
Calidad de la información & 4.353 & 4.444 & 0.285 \\
Calidad del SI & 4.263 & 4.330 & 0.249 \\
Calidad de los servicios & 3.954 & 4.000 & 0.818 \\
Desempeño individual & 4.096 & 4.900 & 0.317 \\
Desempeño colectivo & 3.701 & 3.571 & 0.579 \\
Satisfacción del usuario final & 4.385 & 4.370 & 0.308 \\
\hline
\end{tabular}

Mediante el software estadístico SPSS, se calcularon la normalidad de las variables, las correlaciones y regresiones. También se analizaron la multicolinearidad y otros requisitos estadísticos previos. Los resultados se muestran en los cuadros 4 a 8 , siguiendo el modelo de D\&M cuyas variables servirán para verificar cuáles están más relacionadas con la satisfacción del usuario final y en qué grado. El cuadro 4 presenta los resultados de las pruebas de normalidad de las variables, basadas en los modelos de Kolmogorov-Smirnov y Shapiro-Wilk, los cuales revelan que sólo los factores predictores uso del SI y rendimiento grupal no siguen una distribución normal. 


\section{Cuadro 4 \\ Tests de normalidad}

\begin{tabular}{lcc}
\cline { 2 - 3 } & Kolmogorov-Smirnov & Shapiro-Wilk \\
\cline { 2 - 3 } & Sig. & Sig. \\
\hline Uso del SI & 0.000 & 0.000 \\
Calidad de la información & 0.190 & 0.059 \\
Calidad del SI & 0.064 & 0.142 \\
Calidad de los servicios & 0.056 & 0.028 \\
Desempeño individual & 0.038 & 0.061 \\
Desempeño grupal & 0.003 & 0.001 \\
Satisfacción del usuario final & 0.078 & 0.055 \\
\hline
\end{tabular}

Después de haber utilizado el método de adición como procedimiento para agregar secuencialmente todos los predictores, uno cada vez, el cuadro 5 muestra los coeficientes estandarizados de beta y el valor de la significación de las seis variables bajo estudio. Los valores de $\mathrm{R}(0.848), \mathrm{R}^{2}(0.720)$ y la significación del cambio en $\mathrm{F}(0.000)$ indican que es el modelo que mejor predice la variable satisfacción del usuario final. Se puede observar cómo la variable calidad de la información tiene la beta más alta (0.726), factor que es el más valorado por el usuario final y el que posee el más alto impacto sobre la satisfacción de éste con un SI. Esto es un hecho que debe ser tenido muy en cuenta por la gerencia, así como por los profesionales de SI durante el desarrollo de los mismos. El segundo valor más alto corresponde a calidad del SI (0.492), seguido por el correspondiente a rendimiento individual (0.403). El valor negativo de beta para la variable uso del SI podría ser el resultado de un efecto causado por otros predictores, incluidos o no en el modelo.

Cuadro 5

\section{Coeficientes de beta}

\begin{tabular}{lrrr}
\cline { 2 - 4 } & $\boldsymbol{\beta}$ & Sig. & B \\
\hline (Constante) & --- & 0.003 & 1.670 \\
Uso del SI & -0.255 & 0.171 & --- \\
Calidad de la información & 0.726 & 0.000 & --- \\
Calidad del SI & 0.492 & 0.002 & --- \\
Calidad de los servicios & 0.233 & 0.004 & --- \\
Desempeño individual & 0.403 & 0.003 & --- \\
Desempeño grupal & 0.035 & 0.030 & --- \\
\hline R (0.848), R ${ }^{2}$ (0.720), Sig. F Change (0.000) & &
\end{tabular}


La ecuación lineal resultante de este modelo es:

$$
y=1.670+\left(\beta_{1} \times x_{a}\right)+\left(\beta_{2} \times x_{b}\right)+\left(\beta_{3} \times x_{c}\right)+\left(\beta_{4} \times x_{d}\right)+\left(\beta_{5} \times x_{e}\right)+\left(\beta_{6} \times x_{f}\right)
$$

En el cuadro 6 se pueden observar las cifras obtenidas en las correlaciones de Pearson. La correlación de los predictores uso del SI y rendimiento grupal con la satisfacción del usuario final carece de significación. Esto está en concordancia con algunos estudios empíricos que sugieren que el factor uso de un SI está implícito en la satisfacción del usuario final (IS World Net, 2010), tendiendo así a excluir dicho factor del análisis. La mayoría de los restantes predictores tiene valores moderados, mostrando independencia entre las variables y un impacto significativo de al menos el $95 \%$.

\section{Cuadro 6}

\section{Correlaciones de Pearson}

\begin{tabular}{|c|c|c|c|c|c|c|c|}
\hline & $\begin{array}{c}\text { Satisfacción } \\
\text { del usuario }\end{array}$ & $\begin{array}{c}\text { Uso del } \\
\text { SI }\end{array}$ & $\begin{array}{l}\text { Calidad de la } \\
\text { información }\end{array}$ & $\begin{array}{c}\text { Calidad } \\
\text { del SI }\end{array}$ & $\begin{array}{l}\text { Calidad de } \\
\text { los servicios }\end{array}$ & $\begin{array}{c}\text { Desempeño } \\
\text { individual }\end{array}$ & $\begin{array}{c}\text { Desempeño } \\
\text { grupal }\end{array}$ \\
\hline \multirow{2}{*}{$\begin{array}{l}\text { Satisfacc. del } \\
\text { usuario final }\end{array}$} & 1 & 0.048 & $0.477 * *$ & $0.476 * *$ & $0.250 *$ & $0.390 * *$ & -0.028 \\
\hline & . & 0.692 & 0.000 & 0.000 & 0.035 & 0.001 & 0.814 \\
\hline \multirow{2}{*}{ Uso del SI } & 0.048 & 1 & $0.099 *$ & 0.359 & $0.729 * *$ & -0.029 & -0.167 \\
\hline & 0.692 & . & 0.414 & 0.074 & 0.000 & 0.807 & 0.164 \\
\hline \multirow{2}{*}{$\begin{array}{l}\text { Calidad de la } \\
\text { información }\end{array}$} & $0.477 * *$ & $0.099^{*}$ & 1 & $0.350 * *$ & 0.102 & $0.377 * *$ & $0.635^{* *}$ \\
\hline & 0.000 & 0.414 & . & 0.002 & 0.399 & 0.001 & 0.000 \\
\hline \multirow{2}{*}{ Calidad del SI } & $0.476^{* *}$ & 0.359 & $0.350 * *$ & 1 & $0.326^{* *}$ & $0.435^{* *}$ & $0.560 * *$ \\
\hline & 0.000 & 0.074 & 0.002 & . & 0.006 & 0.000 & 0.000 \\
\hline \multirow{2}{*}{$\begin{array}{l}\text { Calidad de los } \\
\text { servicios }\end{array}$} & $0.250 *$ & $0.729 * *$ & 0.102 & $0.326^{* *}$ & 1 & 0.102 & -0.023 \\
\hline & 0.035 & 0.000 & 0.399 & 0.006 & . & 0.067 & 0.852 \\
\hline Desempeño & $0.0390 * *$ & -0.029 & $0.377 * *$ & $0.435 * *$ & 0.102 & 1 & $0.261 *$ \\
\hline individual & 0.001 & 0.807 & 0.001 & 0.000 & 0.067 & . & 0.028 \\
\hline Desempeño & -0.028 & -0.167 & $0.635 * *$ & $0.560 * *$ & -0.023 & $0.261 *$ & 1 \\
\hline grupal & 0.814 & 0.164 & 0.000 & 0.000 & 0.852 & 0.028 & . \\
\hline
\end{tabular}

**Correlación significativa al nivel 0.01 (bilateral)

*Correlación significativa al nivel 0.05 (bilateral)

Las pruebas de normalidad mostradas en el cuadro 4, así como las estadísticas de multicolinearidad, como la tolerancia, los factores de inflación de la varianza (FIV), las proporciones de la varianza y los resultados en el cuadro 6 sugieren la exclusión de dos predictores: uso del SI y el rendimiento grupal. La eliminación de predictores en un modelo no se suele recomendar, a menos que la variación en 
el error cuadrático medio (ECM) sea igual o menor a la del modelo original, manteniendo o disminuyendo así la varianza del estimador (Webster, 2004). En este caso la variación del ECM es sólo 1/100, cambiando de 0.161 a 0.171 . También puede observarse en el cuadro 5 que esas dos variables están sobre el límite del $95 \%$ y un nuevo modelo de regresión que las excluye lleva las variables restantes a un nivel de significación del 95\%, mientras que el modelo disminuye su calidad de predicción de 0.720 a tan sólo 0.657 . Como resultado, se ha obtenido un modelo reducido con las variables que se muestra en el cuadro 7.

\section{Cuadro 7}

Coeficientes de Beta en el modelo reducido

\begin{tabular}{lccc}
\cline { 2 - 4 } & $\boldsymbol{\beta}$ & Sig. & B \\
\hline (Constante) & --- & 0.005 & 1.883 \\
Calidad de la información & 0.498 & 0.005 & --- \\
Calidad del SI & 0.313 & 0.003 & --- \\
Calidad de los servicios & 0.226 & 0.009 & --- \\
Desempeño individual & 0.253 & 0.002 & --- \\
\hline
\end{tabular}

$\mathrm{R}(0.811), \mathrm{R}^{2}(0.657)$, Sig. F Change (0.000)

También un valor bajo de F (5.457) en el análisis Anova muestra que no hay relación de los predictores con la variable dependiente, dando así solidez al modelo.

La ecuación lineal correspondiente a este modelo es:

$$
y=1.883+\left(\beta_{1} \times x_{a}\right)+\left(\beta_{2} \times x_{b}\right)+\left(\beta_{3} \times x_{c}\right)+\left(\beta_{4} \times x_{d}\right)
$$

La figura 3 muestra los coeficientes estandarizados de beta para los predictores en el modelo reducido:

\section{Figura 3}

\section{Coeficientes de beta estandarizados}

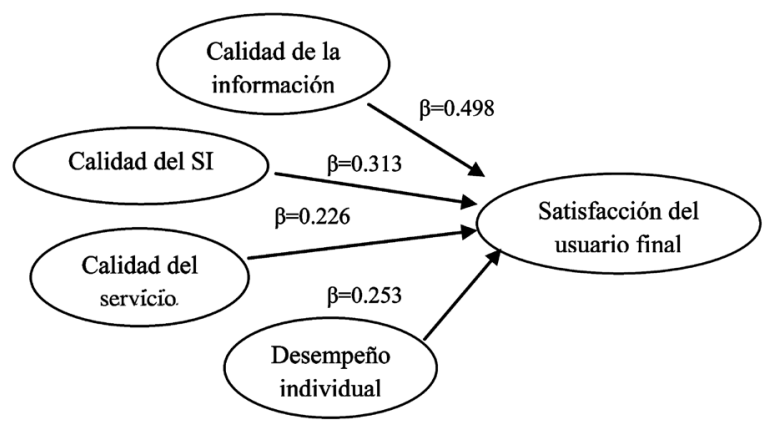


El cuadro 8 muestra los nuevos coeficientes de correlación de Pearson entre las variables en el modelo reducido. Relaciones sólidas moderadas pueden observarse revelando una fuerte correlación entre variables; especialmente entre las predictoras y la dependiente.

\section{Cuadro 8}

Nuevos coeficientes de Pearson

\begin{tabular}{lccccc}
\hline & $\begin{array}{c}\text { Satisfacción } \\
\text { del usuario }\end{array}$ & $\begin{array}{c}\text { Calidad de la } \\
\text { información }\end{array}$ & $\begin{array}{c}\text { Calidad } \\
\text { del SI }\end{array}$ & $\begin{array}{c}\text { Calidad de } \\
\text { los servicios }\end{array}$ & $\begin{array}{c}\text { Desempeño } \\
\text { individual }\end{array}$ \\
\hline Satisfacc. del & 1 & $0.477^{* *}$ & $0.470^{* *}$ & $0.251^{*}$ & $0.384^{* *}$ \\
usuario final &. & 0.000 & 0.000 & 0.005 & 0.002 \\
Calidad de la & $0.477^{* *}$ & 1 & $0.350^{* *}$ & 0.102 & $0.377^{* *}$ \\
información & 0.000 &. & 0.003 & 0.399 & 0.001 \\
& $0.470^{* *}$ & $0.350^{* *}$ & 1 & $0.326^{* *}$ & $0.435^{* *}$ \\
Calidad del SI & 0.000 & 0.003 &. & 0.006 & 0.000 \\
Calidad de los & $0.251^{*}$ & 0.102 & $0.326^{* *}$ & 1 & 0.102 \\
servicios & 0.005 & 0.399 & 0.006 &. & 0.067 \\
Desempeño & $0.0384^{* *}$ & $0.377^{* *}$ & $0.435^{* *}$ & 0.102 & 1 \\
individual & 0.002 & 0.001 & 0.000 & 0.067 &. \\
\hline
\end{tabular}

A partir de los resultados anteriores, se puede observar que todas las variables tienen valores significativos de beta, el cual corresponde a la calidad de la información que es la más significativa estadísticamente. El modelo estadístico original proporciona una varianza explicada del $72 \%$ de las relaciones estadísticas entre estas variables. A pesar de que ese porcentaje disminuye a casi el $66 \%$ en el modelo reducido, el poder de predicción de ambos modelos es relevante para los objetivos de este documento.

En el cuadro 9 se pueden observar los resultados de los niveles de satisfacción de los encuestados, determinado por las respuestas en la muestra a una pregunta directa sobre cómo podría clasificarse su satisfacción con sus SI. Casi el 70\% de los encuestados están por encima de la línea de satisfacción (satisfechos $52.11 \%$ y más que satisfechos $16.9 \%$ ), con sólo el $10 \%$ insatisfechos y el $21 \%$ entre ambos. Un análisis cualitativo posterior, mostrado más adelante en el presente documento, complementa esta información. 
Cuadro 9

Niveles de satisfacción

\begin{tabular}{lcc}
\cline { 2 - 3 } & $\#$ & \% \\
\hline No satisfechos & 7.00 & 9.86 \\
Menos que satisfechos & 15.00 & 21.13 \\
Satisfechos & 37.00 & 52.11 \\
Más que satisfechos & 12.00 & 16.90 \\
\hline Total & 71.00 & 100.00 \\
\hline
\end{tabular}

\section{Procedentes del análisis cualitativo}

Esta sección presenta un resumen de los resultados obtenidos mediante un análisis de los datos cualitativos recolectados en las entrevistas a la alta dirección de las empresas incluidas en la muestra, así como las observaciones al final de los cuestionarios realizadas por los usuarios finales encuestados. El cuadro 10 muestra las cuatro categorías y sus respectivas dimensiones bajo estudio:

\section{Cuadro 10}

\section{Categorías y dimensiones análisis-cualitativo}

\begin{tabular}{lll}
\hline$\#$ & Categorías & \multicolumn{1}{c}{ Dimensiones } \\
\hline 1 & Organización & $\begin{array}{l}\text { Inversión en SI/TI, apoyo organizacional a los SI, arquitectura de } \\
\text { SI, infraestructura, de TI, cultura organizacional }\end{array}$ \\
2 & Calidad & Calidad de la información, calidad del Si, calidad del servicio \\
3 & Desempeño & Individual, organizacional \\
4 & Usuario final & Participación, aportes, uso del Si, satisfacción \\
\hline
\end{tabular}

Con respecto a la infraestructura de TIC, en la categoría organización, estas empresas exhiben un moderado nivel de inversión, donde la mayoría de ellos ha instalado una red de área local (LAN) básica, operada bajo sistema operativo propietario (mayoritariamente una plataforma de Microsoft Windows). Más del 95\% de las empresas no utilizan versiones de software libre o de código abierto de sistemas operativos similares. El uso de computadoras de escritorio por parte de los empleados y directivos de nivel medio, con la asignación de computadoras portátiles para la dirección superior suelen ser el estándar. El acceso a Internet está reservado a los niveles medio y alto, básicamente para acceder al correo electrónico. El número de computadoras personales, impresoras y otros dispositivos periféricos es actua- 
lizado y adecuado a las necesidades del momento. Los servicios de SI y TIC son proporcionados por un contratista externo o por un empleado especialista en estas tecnologías. Conceptos tales como grandes sistemas de gestión de bases de datos (DBMS), intranets, extranets, comercio electrónico o cualquier tipo de e-aplicaciones no han sido implementados. Algunas empresas han desarrollado una página web comercial, estándar, no interactiva.

Las aplicaciones de negocio más utilizadas que se encuentran son los paquetes estándar, aplicaciones de negocios poco o nada integradas, para funciones administrativas y de negocios, especialmente para contabilidad, nómina, recursos humanos, activo fijo, presupuesto, costos, automatización de oficinas y pocos paquetes para diseño ayudado por computadora.

La arquitectura de SI encontrada es simple y se basa principalmente en SI del tipo transaccional con limitado alcance vertical (Laudon y Laudon, 2009). Los desarrollos internos (in-company) de algunas de estas aplicaciones se encuentran sólo en un pequeño número de casos y, básicamente, no se encontraron ninguno de los otros tipos conocidos de SI (ibid.), con la excepción de algunos SI de soporte de decisiones construidos utilizando hojas de cálculo electrónicas. Haciendo una comparación con las etapas de crecimiento de los SI y las TIC del modelo de tres eras de Gottschalk (2002), las pequeñas y medianas empresas estudiadas están en la era 1 (llamada época de procesamiento de datos), la cual se refiere a una etapa básica de procesamiento de datos para lograr eficiencia, donde los datos son el foco y el usuario final tiene que interpretarlos para obtener información. Sólo unas pocas de estas empresas pueden estar en la era 2 (llamada era de TIC), utilizando los SI para lograr la eficacia sobre la base de la información obtenida. Ninguna de estas empresas podría decirse que haya alcanzado la era 3 (llamada era de las redes), caracterizada por el uso de SI estratégicos para lograr la competitividad, donde el foco está en el conocimiento basado en información organizada para apoyar las tareas de conocimiento.

Otro modelo teórico similar es el de Richard Nolan (Cohén y Asín, 2004; Gottsschalk, 2002), el cual se enfoca en el nivel de las inversiones en SI/TIC y consta de nueve etapas o fases de crecimiento de dichas tecnologías. De acuerdo con este modelo, la mayoría de esas empresas analizadas podrían ubicarse en las fases III (control) y IV (integración). 
La etapa III refleja, básicamente, un periodo de control de los gastos en SI y TIC, el uso de SI orientados hacia el control de las operaciones, la planificación y el desarrollo de estándares para los SI, la creación de una unidad de SI y de interfaces automáticas entre los SI. Mientras que la etapa IV es una fase de integración entre los datos y los SI y TIC, adquisición de bases de datos y lenguajes de programación y sustitución de los viejos SI. La mayoría de los directivos de empresas reconocen la necesidad de invertir en SI y TIC.

Sin embargo, afirman estar satisfechos con sus inversiones actuales en estas tecnologías. De hecho, más del $80 \%$ de ellos insisten en no ir más allá de una modesta plataforma que sea suficiente para llevar a cabo sus operaciones rutinarias. También alegaron como razón para esta posición la contracción de las operaciones a las que el sector ha venido enfrentándose desde 2009.

En cuanto a la cultura organizacional de estas empresas, aunque la mayoría de los niveles gerenciales medios y altos promocionan el uso de estas herramientas (y la formación inherente), esto se realiza bajo las limitaciones ya mencionadas. En otros aspectos, la cultura de estas organizaciones no parecen presentar ningún obstáculo para el uso de los SI y TIC.

La mayor parte de las secciones de la guía utilizada para las entrevistas, así como de las encuestas, se basaron en los SI de nómina y recursos humanos, por su complejidad, la relevancia de sus datos y de la información que generan (requerida internamente y por los entes de gobierno relacionados), así como por la necesaria flexibilidad demandada de estos SI.

Aunque los usuarios finales mostraron un grado aceptable de satisfacción con estos SI, hicieron algunas observaciones que reflejan la necesidad de una mayor automatización y conectividad entre los módulos internos y con otros SI. Un beneficio de satisfacer esta necesidad sería reducir significativamente la cantidad de hojas de cálculo electrónicas utilizadas actualmente para preparar informes internos y externos.

Una evaluación cualitativa de la calidad percibida por el usuario final de estas dimensiones analizadas en el modelo cuantitativo como variables o factores, revela que éstas mantienen la misma jerarquía en ambos enfoques. El usuario final asignó gran importancia a la calidad de la información, incluso a expensas de, por ejemplo, un SI lento, una interfaz de usuario final poco amigable, fallos técnicos o un 
soporte deficiente por el personal del departamento o unidad de SI. Esto coloca la importancia de la calidad técnica de los SI y de los servicios informáticos de apoyo en segundo y tercer lugares, respectivamente, y es fundamental para la gerencia y los profesionales de SI durante el desarrollo o la adquisición de un SI, como un recordatorio de dónde debería centrarse el principal foco de atención.

Como un beneficio final de un SI, el usuario final espera que éste sea un soporte que le permita aumentar su rendimiento laboral. Además, la gerencia espera que esto, por acumulación, se transforme en un mayor y mejor desempeño organizacional. A este respecto, el usuario final manifestó sentir un incremento en su rendimiento, pero mostró un deseo por una mejor funcionalidad de los SI. También, aunque la alta dirección considera aceptable el rendimiento a nivel de grupo, han señalado que hay margen para mejorar.

Por último, el usuario final mostró satisfacción con su participación en el desarrollo (o en su evaluación, en caso de compra) del SI, así como con su contribución a la implementación del mismo. El hecho de ser las pequeñas y medianas empresas organizaciones con relativamente poco personal les permite mayor eficiencia y control de sus procesos. Finalmente, al igual que en el análisis cuantitativo, el usuario final manifestó un nivel aceptable de satisfacción con el SI.

\section{Conclusiones}

Una primera conclusión se centra en que los problemas encontrados referentes al tratamiento de la información no obedecen a una falta de satisfacción del usuario final con sus sistemas de información automatizados. Esta satisfacción en ambos análisis, cuantitativo y cualitativo, resultó aceptable y los dos principales factores que la afectan son la calidad de la información y la calidad de los SI. La primera tiene procedencia sobre todos los demás factores en el modelo de D\&M, aparentemente confirmando la presunción de que las deficiencias de algunos de ellos podrían ser ignoradas, siempre y cuando la información entregada cumpla con los criterios de calidad de información esperados por el usuario final. Sin embargo, la satisfacción del usuario final es algo relativa, debido a que las necesidades de información en este tipo de negocios no suelen alcanzar niveles de cantidad o complejidad altos y también debido a ciertas quejas planteadas por los usuarios finales encuestados. Dada la utilización casi exclusiva de paquetes estándar de SI, los cuales suelen ser rígidos por naturaleza, cabe esperar algunas deficiencias de información. Estos hallazgos son favorables, teniendo en cuenta que los SI de 
nómina y recursos humanos contribuyen fuertemente a la información demandada, tanto interna como externamente, y que es útil para el control y el desarrollo de los recursos humanos de una organización. Lo anterior da respuesta a nuestra pregunta de investigación. El modelo de D\&M ha sido de gran utilidad para la realización de este estudio.

Una conclusión importante es que más del $85 \%$ de los usuarios finales reveló que necesitan recurrir a software de hojas electrónicas para complementar los datos faltantes o preparar informes. Aunque esta situación es normalmente considerada como una importante deficiencia en un SI, en el caso de paquetes de software estándar el usuario final parece aceptarla. No obstante, esto podría ser una razón de por qué, en no pocos casos, el usuario final ha indicado que le gustaría tener más funcionalidad en sus SI.

En la práctica, los niveles gerenciales y el personal de informática tienden a veces a poner más atención en aspectos de forma o en las características técnicas de un SI, en lugar de ponerla en los aspectos sustanciales o de fondo. El costo atractivo de un SI también puede contribuir a pasar por alto estos últimos aspectos. El usuario final está generalmente dispuesto a renunciar a ciertos atributos, tales como la facilidad de uso, la velocidad de procesamiento o una interfaz de usuario final atractiva, a cambio de una información pertinente y de calidad.

Una ventaja de la Pyme de tamaño medio sobre las pequeñas es su mayor capacidad para invertir en SI y TIC. Éste es el caso de la mayoría de las empresas estudiadas, lo cual permite explicar una posible contradicción con lo expresado en la introducción con respecto a las pocas inversiones, y la calidad de las mismas, que este tipo de organizaciones realizan en SI y TIC. Igualmente, aun cuando pudiesen estar en etapas más avanzadas, estas pequeñas y medianas empresas ponen mayor interés en la gestión de la información y en el suministro de la misma a los entes oficiales que la demanden.

La ausencia de Sistemas de Información Estratégicos (SIS), e-business y Sistemas de Información para Ejecutivos (EIS) representa una pérdida de oportunidades para el crecimiento económico de las empresas estudiadas. La alta gerencia de la Pyme debería diseñar una estrategia para iniciar un uso efectivo de la Internet como medio para llevar a cabo diferentes modalidades de transacciones electrónicas tales como algunos tipos de e-commerce: Business to Business (B2B), Business to Customers (B2C) y Business to Government (B2G). Estas modalidades de SI basados 
en la Web y orientados a fortalecer las relaciones con los clientes y proveedores se consideran hoy como activo indispensable. Un elemento positivo es que la cultura organizacional en la mayoría de estas empresas parece ser favorable a este tipo de cambio. No obstante, los directivos deben tener siempre presente que el hecho de invertir más que sus competidores en tecnologías de la información no necesariamente dará a sus empresas una ventaja significativa sobre estos últimos. Será la gestión de dichas tecnologías lo que podrá marcar la diferencia.

Las universidades tienen un nicho de oportunidades en el sector de la Pyme para la realización de proyectos prácticos de SI y TIC, con lo cual pueden contribuir muy positivamente al diseño de mejores prácticas de gestión. Estas acciones deben reforzar las pequeñas y medianas industrias para alcanzar una nueva etapa de crecimiento de sus herramientas de tecnologías de la información.

\section{Referencias}

Aimm (2009). Capítulo Guayana. Relación de industrias metalmecánicas de ciudad Guayana. Asociación de industrias metalmecánicas y metalúrgicas, estado Bolívar.

Bailey, J. y S. Pearson (1986). Development of a tool for measuring and analyzing computer user satisfaction. Management science (29): 530-545.

Cohén, D. y E. Asín (2004). Sistemas de información para los negocios. 4ª ed., México: McGraw Hill Interamericana.

Cornella, A. (1997). Los recursos de información. Ventaja competitiva de las empresas. Madrid: McGraw Hill Interamericana de España.

DeLone, W. y E. Mclean (2003). The DeLone and Mclean model of information systems success: A ten-year update. Journal of Management Information Systems (19): 9-30.

(1992). Information systems success: The quest for the dependent variable. Information Systems Research 3 (1): 60-95.

Doll, W. y G. Torkzadeh (1998). Developing a multidimensional measure of systemuse in an organizational context. Information \& Management (33): 171-185. 
Doyle, D. (2009). European SME facts and figures - Small business impact in Europe. The Institute of Certified Bookkeepers. Disponible: http://www.bookkeepers.org.uk/News/566.

E-encuesta. (2010) Customer satisfaction. Disponible: http://www.e-encuesta. com/previewTemplates.do.

European Commission (2010). Enterprise and industry - small and medium-sized enterprises. Disponible: http://ec.europa.eu/enterprise/policies/sme/factsfigures-analysis/.

Gottsschalk, P. (2002). Toward a model of growth stages for knowledge management technology... Informing Science 5 (2): 79-93.

Hummel, B. (2001). Indicadores del impacto tecnológico en las comunidades. Documento nuevas tecnologías de la información para el desarrollo loca. El proyecto Adapt-Nutrias del suroccidente de Asturias. Gijón: Ediciones Trea, S.L.

INE (2009). Listado y clasificación de las industrias metalmecánicas de ciudad Guayana. Instituto Nacional de Estadísticas. Estado Bolívar, Venezuela.

Is World Net (2010). Information systems effectiveness. Disponible: http://business.clemson.edu/ISE/html/information_quality.html.

Jiang, J., G. Klein y C. Carr (2002). Measuring information system service quality: SERVQUAL from the other side. MIS Quarterly 26 (2): 145-166.

, J. Roan y J. Lin (2001). Is service performance: self- user perceptions. Information \& Management 38 (8): 499-506.

Kotler, P., G. Armstrong, D. Cámara e I. Cruz (2004). Marketing. 10a. ed., Madrid: Prentice Hall. Pearson Education.

Laudon, K. y J. Laudon (2009). Management Information Systems. New Jersey: Pearson Education Inc. 
Mahmood, M., J. Burn, L. Gemoets y C. Jacquez (2000). Variables affecting information technology end-user satisfaction: A meta-analysis of the empirical literature. International Journal of Human Computer Studies 52 (4): 751771.

Mahmood, M.A., L. Hall y D.L.Swanberg (2001). Factors affecting information technology usage: A meta analysis of the empirical literature. Journal of Organizational Computing and Electronic Commerce 11 (2): 117-130.

Medina, M. (2005). Evaluación del impacto de los sistemas de información en el desempeño individual del usuario. Aplicación en instituciones universitarias. Tesis doctoral. Universidad Politécnica de Madrid.

Myers, M. (2007). Qualitative research in information systems. Versión modificada. Disponible: http://www.qual.auckland.ac.nz/.

(2009). Qualitative research in business and management. Townbridge: Cromwell Press Ltd.

(2010). Qualitative research in information systems. Versión modificada. Disponible: http://www.qual.auckland.ac.nz/.

PDESN (2007). Plan de desarrollo económico y social de la nación. 2007-2013. Caracas: Ministerio del poder popular para la comunicación e información. Disponible: http://www.embavenez-paris.com/docs/lineas_gen_nacion.pdf.

Pitt, L., R. Watson y B. Kavan (1995). Service quality: A measure of information systems effectiveness, MIS Quarterly 19 (2): 173-188.

PNCTI (2010). Plan nacional de ciencia, tecnología e innovación, 2005-2030. Caracas: Ministerio de ciencia y tecnología. Disponible: www.fonacit.gov. ve/documentos/pncti.pdf.

Rai, A., S.S. Lang y R.B. Welker (2002). Assessing the validity of IS success models: An empirical test and theoretical analysis. Information Systems Research 13 (1): 50-69. 
Saarinen, T. (1996). An expanded instrument for evaluating information system success, Information \& Management (31): 103-118.

Shin, B. (2002). A case of data warehousing project management. Information \& Management 39 (7): 581-592.

Torkzadeh, G. y J. Lee (2003). Measures of perceived end-user computing skills. Information \& Management 40 (7): 607-615

Torkzadeh, G. y W. Doll (1999). The development of a tool for measuring the perceived impact of information technology on work. The International Journal of Management Science 27 (3): 327-339.

Universia knowledge@wharton (2010). The SMEs multiple challenges in Latin America. Universia Colombia. Disponible: http://www.universia.net.co/ laboral-empresarial/destacado/los-multiples-retos-de-las-pymes-en-america-latina.html.

Webster, A. (2004). Estadística aplicada a los negocios y la economía. Bogotá: McGraw Hill Interamericana.

Wixom, B.H. y P.A. Todd (2005). A theoretical integration of user satisfaction and technology acceptance, Information Systems Research 16 (1): 85-102. 